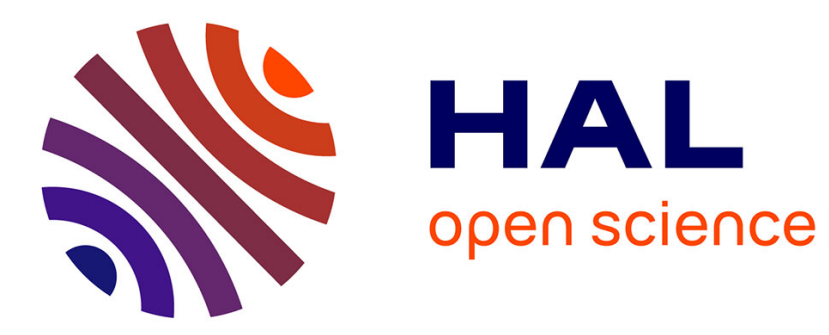

\title{
Sortie d'un placement à long terme et modes d'accès à l'indépendance
}

\author{
Monique Crost, Pascale Donati, Annick-Camille Dumaret
}

\section{To cite this version:}

Monique Crost, Pascale Donati, Annick-Camille Dumaret. Sortie d'un placement à long terme et modes d'accès à l'indépendance. Revue internationale de l'éducation familiale, 2009, 26, pp.15-34. inserm-00469584

\section{HAL Id: inserm-00469584 https://www.hal.inserm.fr/inserm-00469584}

Submitted on 1 Apr 2010

HAL is a multi-disciplinary open access archive for the deposit and dissemination of scientific research documents, whether they are published or not. The documents may come from teaching and research institutions in France or abroad, or from public or private research centers.
L'archive ouverte pluridisciplinaire HAL, est destinée au dépôt et à la diffusion de documents scientifiques de niveau recherche, publiés ou non, émanant des établissements d'enseignement et de recherche français ou étrangers, des laboratoires publics ou privés. 


\title{
Sortie d'un placement à long terme et modes d'accès à l'indépendance
}

\author{
M. Crost, P. Donati, A.C. Dumaret ${ }^{1}$
}

\begin{abstract}
Résumé
Cet article explore les principales étapes conduisant à l'indépendance de 123 jeunes placés avec leur fratrie en villages d'enfants et sortis entre 1972-2001. Les jeunes générations d'« anciens placés » ont bénéficié de plus d'aides institutionnelles mais ont été confrontées à une entrée plus difficile sur le marché du travail. Au total, ce placement stable ( $\mathrm{m}=9$ ans) a permis à $60 \%$ des jeunes d'obtenir un diplôme et à $65 \%$ d'acquérir leur indépendance financière et résidentielle à l'âge de 24-25 ans sans difficultés majeures. La solidarité fraternelle et le support des mères SOS ont facilité cette période de transition. L'accès à cette indépendance est très lié à l'obtention de diplômes quel qu'en soit le niveau et à l'absence de graves problèmes psychiques ou comportementaux à la sortie du placement. Pour ces jeunes, comme pour tous ceux de la Protection de l'enfance, la prolongation des accompagnements plusieurs années après leur sortie s'avère non seulement indispensable mais doit être développée pour favoriser au mieux l'entrée dans la vie adulte.
\end{abstract}

Mots-clés : Protection de l'enfance, villages d'enfants, prises en charge, diplômes, troubles comportementaux, santé mentale, support socio-familial, accès à l'indépendance.

\section{Summary}

This article explores the principal stages leading to independence of 123 youths who were reared with their siblings in Children's Villages and who left care between 1972-2001. The youngest generations of ex-care have received more care services but had more difficulties to enter the labour market. This stable placement (mean of duration=9 years) allowed $60 \%$ of them to obtain a diploma and $65 \%$ to become financial and housing independent without major difficulties by the age of 24-25. Family solidarity among siblings and the support of SOS mothers were pivotal during this period. Access to independence is linked to diploma, whatever the level, and to the absence of severe mental health problems when leaving care. For these youths, as for all youths leaving the Child Welfare services, prolonging case worker support by several years prove to be vital and must be developed to help them make successful transition to adulthood.

Key words: Child Welfare, children's villages, leaving care, education, behaviour, mental health, social support, transition to adulthood.

\footnotetext{
${ }^{1}$ CERMES Centre de recherche médecine, sciences, santé et société - INSERM U750, Université Paris Sud Paris XI - EHESS, 7 rue Guy Môquet, 94801 Villejuif cedex, France Auteur correspondant : Dumaret A.C., Adresse e-mail : dumaret@vjf.cnrs.fr
} 


\section{Introduction}

Le moment de la sortie d'un placement à l'Aide sociale à l'enfance, en famille d'accueil ou en institution, signifie la cessation de la prise en charge et de l'étayage socio-éducatif. Cette étape de transition s'avère difficile pour les jeunes puisqu'elle représente souvent la séparation avec le seul milieu environnemental et familial stable qu'ils aient connu. C'est le cas pour ceux qui ont été accueillis avec leurs frères et sœurs pendant une longue période en villages d'enfants. Dans le cadre d'une recherche sur leur devenir à long terme, une analyse spécifique a porté sur la phase transitionnelle vécue entre la fin des prises en charge jusqu'à l'entrée dans la vie adulte, à l'âge de 25 ans. Grâce à une observation menée sur trois décennies, c'est à la fois l'évolution des prises en charge au sein de l'institution et les transformations générales du contexte socio-économique qui ont été prises en compte. Cet article se centre sur les conditions de sortie du placement et les principales étapes des cheminements individuels selon les ressources personnelles et les soutiens institutionnels et familiaux dont ces jeunes ont bénéficié.

\section{Revue de la littérature}

Depuis une vingtaine d'années, de nombreux travaux qualitatifs anglo-saxons se sont centrés sur le moment succédant la fin de la prise en charge, dans le cadre de projets de révision de lois sur la Protection de l'enfance. Plus rares, les études sur le devenir à long terme se heurtent à la difficulté de retrouver ces adultes, anciennement placés, et sont soumises au biais de mémoire des enquêtés qui peuvent avoir oublié ou veulent occulter cette période de leur vie. Si cette grande diversité des approches et des populations d'étude limite la comparabilité des résultats (Frechon \& Dumaret, 2008), on retient toutefois que la sortie du placement est mieux vécue pour les jeunes élevés en familles d'accueil que pour ceux élevés en foyers.

Plus généralement, le vécu du passage à l'âge adulte est plus difficile pour ces jeunes anciennement placés que pour leurs pairs, et cela, dans tous les domaines de la vie, sociale, professionnelle et personnelle. Plus difficile, cette phase de transition se différencie aussi de celle vécue par les autres jeunes du même âge par une accélération des étapes sous la contrainte d'une limitation temporelle du processus d'insertion socioprofessionnelle, en l'absence de soutien de la part de la famille d'origine ${ }^{2}$ (Goyette, 2006 ; Stein, 2006 ; Stein \& Munro, 2008). Aussi est mis en avant le poids déterminant de la scolarité et de la formation professionnelle dans le futur parcours des sortants. Nombre d'études montrent l'importance des retards scolaires : selon Pecora et col. (2006), les 16-24 ans sans diplôme ou hors scolarité et sans emploi sont plus nombreux que dans la population générale. Courtney \& Dworsky (2006) indiquent que plus d'un tiers n'ont aucun diplôme et qu'ils sont beaucoup plus nombreux que leurs pairs à être exclus de l'école et du travail (32\% vs 12\%). Wade \& Dixon (2006) ainsi que Courtney, Piliavin, Grogan-Kaylor \& Nesmith (2001) montrent l'existence d'un lien entre échecs scolaires, emplois peu qualifiés et faible rémunération, comme l'avaient déjà montré des recherches plus anciennes (Stein, 1994). En France où les travaux sur la sortie du placement étaient jusque récemment très peu nombreux, on retient l'étude de Corbillon, Dulery \& Mackiewicz (1997) portant sur 62 jeunes ayant quitté un foyer, les plus âgés ayant 26 ans ; si 43\% ont un travail, il s'agit généralement d'emplois sans qualification.

\footnotetext{
${ }^{2}$ Stéphanie Gaudet par exemple montre combien, pour la jeunesse d'aujourd'hui, « l'aide reçue par la famille est un des éléments qui influencent le plus les parcours d'entrée dans l'âge adulte » (2007, p. 15).
} 
Conditionnée par l'autosuffisance financière et donc l'emploi, la question du logement est posée comme une des plus grandes difficultés pour les jeunes à la sortie des placements. L'étude espagnole de Del Valle et col. (2008) montre qu'en cas de sortie précoce, quatre jeunes sur dix retournent dans leur famille. Dans plusieurs études américaines et anglaises précitées, les auteurs soulignent un pourcentage élevé de «homeless » (10-30\%). L'instabilité est partout soulignée, en particulier, pour la France avec l'étude de Frechon (2005) sur les trajectoires résidentielles de femmes de 19 à 32 ans où de multiples déménagements ont lieu dans les trois premières années après la sortie ; données confirmées par une étude en cours du CNFE-PJJ (Hubert, Tournyol du Clos, Cosio \& Frechon, 2006). Les instabilités personnelles et affectives, les difficultés dans la vie relationnelle ainsi que la faiblesse du réseau amical sont souvent notées. La plupart des jeunes font état d'un sentiment d'abandon à la sortie, ce qui conduit à s'engager rapidement dans la vie conjugale, laquelle constitue un relais à des relations familiales insatisfaisantes (Corbillon, Dulery \& Mackiewicz, 1997 ; Frechon, 2005). Est soulignée la fréquence des grossesses précoces et des IVG, notamment dans les milieux socioculturels très défavorisés (Rutter, Quinton \& Hill, 1990 ; Carpenter, Clyman, Davidson \& Steiner, 2001). Les problèmes de santé mentale, quant à eux, ont été étudiés à travers leur impact sur l'insertion professionnelle et l'accès au logement individuel (Pinkerton \& Stein, 1995 ; Rashid, 2004 ; Lenz-Rashid, 2008). Ces problèmes, tels que dépression, tentatives de suicide, stress post-traumatiques, abus ou dépendance à l'alcool ou autres substances, varient de 25 à 40\% parmi les sortants âgés de 20-25 ans (Andersson, 2005 ; Wade \& Dixon, 2006), les taux les plus élevés concernent les plus jeunes d'entre eux et ceux multiplacés.

Quels que soient les pays, les études, surtout celles à plus long terme, soulignent la fréquence des problèmes avec la justice et des suivis par des équipes sociales, de 25 à $35 \%$, problèmes plus fréquemment observés parmi les hommes que les femmes et centrés autour du moment de la sortie (Festinger, 1983 ; Bauer, Dubechot \& Legros, 1993 ; Coppel \& Dumaret, 1995 ; Dumaret, Coppel \& Couraud, 1997 ; Del Valle, Bravo, Alvarez \& Fernanz, 2008). Au final, le consensus qui se dégage est la nécessité de préparer cette période délicate de la sortie très tôt pendant le placement (Reid \& Dudding, 2006 ; Storø, 2008), et de prolonger les soutiens et accompagnements au-delà de la fin des prises en charge, notamment pour les jeunes les plus en difficultés.

\section{Contexte institutionnel}

Créée en 1956, l'association «SOS Villages d'enfants » répond à la situation d'enfants sans parents ou en grandes difficultés dans leur milieu, leur apportant par sa structure : «une maison, une mère SOS dans un village », bien-être, affection et éducation. Sa spécificité est l'accueil de fratries sur une longue durée ${ }^{3}$. L'association gère aujourd'hui douze villages d'enfants (VE) en France métropolitaine, agréés par l'Aide sociale à l'enfance (ASE) et bénéficiant généralement d'une habilitation justice (Protection judiciaire de la jeunesse, PJJ). L'un d'eux, terrain de la recherche, accueille des enfants au sein de dix maisons familiales, chaque maison étant placée sous la responsabilité d'une «mère SOS ». Ce village d'enfants, créé il y a un peu plus de trente ans, a vu son équipe psycho-éducative se structurer progressivement dans le milieu des années 1980 et se dessiner de nouveaux profils d'enfants accueillis (fratries non orphelines, sorties ou réorientations avant l'âge de la majorité). Cette période témoigne aussi des transformations dans le fonctionnement de l'institution : plus grande exigence de formation pour les directeurs de village, accueil de plusieurs fratries

\footnotetext{
${ }^{3}$ Cette structure correspond à des maisons d'enfants à caractère social (MECS). L'association est rattachée à SOS Children's villages International, ONG composée de 100 villages d'enfants en Europe.
} 
cohabitantes dans une même maison, professionnalisation des mères SOS dont le statut d'éducatrices familiales sera reconnu en janvier 2005.

C'est en 2003 que cette institution, ayant le souci d'évaluer la qualité de ses prises en charge, a émis le souhait qu'une recherche soit réalisée sur le devenir adulte des jeunes placés. Un premier article a étudié les relations entre facteurs de risque et troubles comportementaux et psychiques des jeunes, notamment la violence intra-familale, la pathologie psychiatrique parentale et la gravité des négligences et maltraitances (Dumaret \& Crost, 2008). Le présent article, fondé sur une analyse quantitative, se centre sur la période allant de la sortie du placement à la vie autonome vers l'âge de 24-25 ans.

\section{Matériel et méthode}

La méthodologie utilisée est celle d'une approche longitudinale dans le passé : les sujets ont été sélectionnés sur la base d'archives puis localisés et évalués ultérieurement. Le protocole de recherche a reçu l'aval de la CNIL en 2004. Un recensement exhaustif des admissions jusqu'en 2000 a montré que 150 jeunes avaient quitté l'institution à 15 ans et plus. Parmi eux, 130 font partie de l'étude, répondant aux trois critères suivants : avoir été placés pendant au moins trois ans, être sortis depuis au moins trois ans, et être âgés d'au moins 23 ans à l'enquête. Ils ont été admis avant 1992 (certains avaient transité avec leur mère SOS par un autre village d'enfants), les sorties se sont étalées de 1972 à 2001. Aujourd'hui, ces personnes, autant d'hommes que de femmes, ont entre 23 et 50 ans (âge médian : 38 ans).

L'analyse est issue de l'étude des dossiers institutionnels et d'une enquête postale. Celle-ci réalisée au moyen de questionnaires (dont l'un standardisé sur la santé et l'autre construit pour l'enquête) et effectuée auprès des 123 anciens placés (sept sont décédés), visait à connaître, outre la situation actuelle des «anciens placés », les conditions de vie dans la période qui suivit la sortie (hébergement, aides et personnes ressources...). Un important taux de réponses a été obtenu : 99 anciens placés ont renvoyé leurs questionnaires (80\%).

Des informations complémentaires ont été recueillies auprès des membres de l'équipe socio-éducative (leurs 22 mères SOS, 2 directeurs de VE). Ainsi, un matériel très important a été recueilli à partir de plusieurs sources de données. Le fait que l'institution dispose depuis très longtemps de fiches de synthèse retraçant l'évolution annuelle de l'enfant (scolarité, comportement) a permis de repérer les principaux événements lors du placement et après. Les entretiens avec les anciens placés et ceux qui les avaient eus en charge ont permis de préciser et croiser les données du questionnaire postal: a) type de difficultés rencontrées à la sortie (démêlés avec la justice...), b) solidarités familiales, c) chronologie et modalités d'insertion.

Les indicateurs des conditions de sortie et d'accès à l'indépendance sont les suivants :

- âges d'admission et de sortie : les dates de sorties physiques du lieu de placement ont été distinguées de celles des sorties par fin de prises en charge administratives et financières.

- les difficultés psychiques et troubles à l'adolescence et/ou au moment de la sortie (in late adolescence) : ils n'ont pas fait l'objet de recueil par questionnaires standardisés. Les dossiers institutionnels ont fait l'objet d'une analyse de contenu, complétée par les observations de l'équipe. Les troubles ont été hiérarchisés en fonction de leur gravité (Dumaret \& Crost, 2008).

- aides au moment de la sortie et ultérieurement : soutiens matériels et relationnels, aides scolaires et à l'insertion professionnelle (chantier école...), aides administratives (recherche de logement ou d'emploi...).

- le niveau scolaire atteint et les diplômes obtenus.

- l'insertion socioprofessionnelle et l'indépendance résidentielle. On considère l'indépendance dans le sens d'une autosuffisance sur le plan matériel et financier à l'âge de 24-25 ans ; la notion d'autonomie renvoyant davantage aux capacités personnelles à orienter sa vie et faire 
ses propres choix. Les processus d'insertion étudiés repèrent les étapes franchies en terme de : a) formation, b) travail en distinguant les petits boulots d'un emploi stable (pendant au moins 6 mois), c) mise en union, et d) accès à un logement indépendant. Pour les hommes, ont été retenus les hébergements et logements après le service militaire. L'ensemble des données recueillies a permis d'évaluer l'accès à l'indépendance financière et résidentielle de 114 de ces adultes en tenant compte des difficultés de parcours : indépendance acquise avec ou sans problèmes majeurs (aucune aide institutionnelle pour tous), pas d'indépendance.

Les 123 sujets appartiennent à 37 familles mais tous n'ont pas eu le même parcours avant le placement en VE. Aussi, les analyses ont été effectuées sur les situations individuelles et les facteurs de risque propres à chacun. Trois classes d'âge ont été distinguées : les sujets âgés de 42-50 ans à l'enquête, les sujets de 34 à 41 ans, et ceux de moins de 34 ans, afin de considérer trois générations de sortants très différenciées. Les plus anciennes sont sorties avant 1980 et les plus jeunes générations à partir de 1990.

Les analyses statistiques ont été effectuées à l'aide du logiciel SAS (version 9.1); les différences significatives sont mises en évidence à l'aide des tests de Chi2 tenant compte de la puissance de l'échantillon.

\section{Résultats}

\section{Présentation de la population d'étude et situation à la sortie}

Violences familiales, négligences graves et précarité sociale prédominent dans les milieux d'origine des enfants, même si le décès parental a été longtemps la principale cause d'admission. Avant leur arrivée, $36 \%$ des jeunes ont subi des maltraitances et un quart d'entre eux ont connu au moins deux autres placements; la moitié avaient des retards scolaires importants. Si quatre sur dix n'ont vécu qu'avec leur fratrie et leur mère d'accueil, les autres ont " cohabité » avec d'autres fratries et quelques uns ont successivement été élevés par deux mères SOS. Malgré l'importance de l'étayage durant le placement (rééducations, suivis psychologiques, activités extra-scolaires...), 42\% des adolescents présentent toujours des troubles comportementaux et psychologiques à la sortie, plus souvent les garçons que les filles.

Dans plus d'un cas sur quatre, la prise en charge a duré 13 ans. Le mode de sortie dominant est la fin de prise en charge à la majorité. Avec le temps, les prises en charge, incluant depuis 1974 les contrats aux Jeunes Majeurs ${ }^{4}$, ont été prolongées après l'âge de la majorité (19-21 ans).

Tableau 1 certaines questions totalisent moins de 123 réponses

Les sorties précoces du VE avant l'âge de la majorité à 18 ans sont significativement corrélées à l'existence de problèmes psychiques et comportementaux importants (comportements pré-délinquants et violents, abus d'alcool parmi les hommes, fugues pour les filles). Ces sorties, qui renvoient à une époque où l'équipe éducative n'existait pas, ont concerné des jeunes admis à un âge relativement élevé (8-13 ans) : a) retours dans la famille d'origine dont la majorité se sont soldés par de nouvelles prises en charge par l'ASE, b) réorientations en internat d'aînés de fratrie afin de préserver les autres frères et sœurs et la mère SOS, c) départs anticipés à l'armée pour les hommes et/ou foyer et mise en couple rapide pour les femmes. Les sorties tardives à 19 ans et plus témoignent, pour un tiers des jeunes, de difficultés psychologiques de l'ordre de l'anxiété, de la dépression, avec une

\footnotetext{
${ }^{4}$ In 2007, on dénombrait 16,600 Jeunes Majeurs, parmi 141,000 jeunes accueillis à l'Aide sociale à l'enfance (Bailleau, G., Trespeux, 2008).
} 
grande immaturité affective. Ces prises en charge prolongées ont certainement constitué une protection pour des jeunes porteurs d'un handicap physique ou intellectuel.

Au moment de la sortie du placement, des aides importantes et diversifiées ont été apportées par l'institution à plus de la moitié des jeunes, bénéficiant surtout aux jeunes générations de sortants (figure 1). Leur prolongation au-delà de la majorité, dont les contrats Jeunes Majeurs, a permis l'achèvement des scolarités et l'acquisition de diplômes.

Figure 1

Un chantier-école a permis à certains, surtout les plus en difficultés, d'avoir un minimum de formation professionnelle, soutien auquel ont succédé d'autres formes d'accompagnement par des éducateurs (service aux aînés pour la recherche de logement ou d'emploi...) : 73\% des plus jeunes générations en ont bénéficié vs $10 \%$ des plus âgées, en particulier les hommes en difficultés et lors du retour du service militaire pour les générations médianes $(\mathrm{p}<0,001)$. En revanche, quelle que soit l'époque du placement, un cinquième des jeunes ont reçu une aide financière par l'institution ou par des fondations privées. De manière générale, ce sont les aides axées sur le logement qui ont été les plus fréquentes : appartements relais, aides financières et/ou hébergement par la mère SOS.

Diplômes obtenus et problèmes de santé mentale

Quatre jeunes sur 10 ont quitté le cursus scolaire sans diplôme, presque trois fois plus que dans la population nationale (15\%, INSEE). En revanche, la part de ceux ayant des diplômes non qualifiants est similaire à celle des autres jeunes Français $(16 \%)$, c'est le cas aussi pour les CAP-BEP, $28 \%$ vs $31 \%$ ). Ce dernier constat reflète les préoccupations de ceux qui ont en charge des jeunes de la protection de l'enfance, tendant à favoriser la formation professionnelle en vue d'une insertion rapide dans la vie active. C'est ainsi que l'on ne trouve que $15 \%$ d'anciens placés munis d'un diplôme équivalent ou supérieur au baccalauréat, alors qu'ils sont $38 \%$ dans la population française. On trouve autant d'adultes sans diplôme dans les deux groupes d'âge extrêmes, mais ce sont les générations intermédiaires qui sont les moins diplômées $(55 \%)$.

\section{Figure 2}

Les principaux obstacles que rencontrent ces jeunes, comme tous ceux de l'ASE, renvoient à une double problématique: des difficultés psychologiques et une origine sociale défavorisée $e^{5}$. Les sujets qui présentaient des troubles psychologiques et comportementaux graves à l'adolescence ou à la sortie étaient nettement plus souvent des garçons et des jeunes déjà en échec scolaire à l'entrée du secondaire. Ils étaient au moins deux fois plus nombreux à ne pas avoir de diplôme (ou de bas niveau) que les jeunes sans troubles notoires. Si l'impact des difficultés comportementales sur la scolarité est bien connu, on se doit toutefois de noter que la gravité des troubles n'empêche pas tous les jeunes de suivre une formation ( $37 \%$ ont un niveau CAP-BEP, $15 \%$ un niveau bac) ; en revanche, elle diminue leurs chances d'accéder au diplôme (respectivement : $26 \%$ et $7 \%$ ).

\section{Modalités d'accès à l'indépendance}

Indépendamment des problèmes de comportement ou de santé mentale, on peut considérer qu'aux alentours de 24-25 ans, $65 \%$ des jeunes ont acquis leur indépendance sans problème majeur : un logement individuel et une sécurité financière, notamment les plus âgés et les femmes. Pour $24 \%$, les parcours se sont avérés beaucoup plus difficiles (surtout pour les

\footnotetext{
${ }^{5}$ Une étude sur «Les trajectoires scolaires des enfants 'pauvres' » souligne la manière dont les parcours se différencient précocement : les écarts se creusent dès l'entrée en sixième (Davaillon \& Nauze-Fichet, 2004).
} 
générations intermédiaires) et pour $11 \%$, n'ont abouti à aucune forme d'indépendance. Rappelons que l'indépendance est plus tardive pour les plus jeunes, qui par ailleurs n'ont pas tous eu le temps d'en franchir toutes les étapes.

\section{Chronologie des étapes et insertion socioprofessionnelle des jeunes}

La succession des étapes reflète les modèles sexuellement différenciés d'entrée dans l'âge adulte avec, pour les hommes, le préalable d'une insertion professionnelle à la formation du premier couple (Tableau 1). Le moment de l'entrée dans la conjugalité est plus précoce pour les femmes. En revanche, si les femmes sont nombreuses à se conformer au modèle ancien d'une union précoce sans installation professionnelle, trois fois plus souvent que les hommes, beaucoup connaissent une séquence de vie professionnelle avant de construire un couple. Notons que très peu de femmes ont eu leur premier enfant avant 20 ans (14\%), à la différence de ce qui est mentionné dans la plupart des travaux anglo-saxons. Pour les femmes, l'âge moyen au premier enfant est de 23 ans, $\sigma=4$ et la médiane de 26 ans $(n=43)$.

Pour les hommes, deux fois plus souvent que pour les femmes, l'expérience d'une succession de petits emplois précède la stabilité d'un emploi durable, d'autant plus en l'absence de diplôme. Alors qu'en France, «l'avantage que confère la possession d'un diplôme pour l'accès au marché de l'emploi, ne cesse de se renforcer » (Caille, 2003, p.180), elle est une nécessité essentielle pour les sortants des placements. Ainsi, quand l'insertion commence par un emploi, avant la mise en couple et le logement, il s'agit majoritairement de jeunes diplômés, en proportion identique de l'enseignement général ou professionnel.

\section{Le logement indépendant : témoin d'une indépendance matérielle avérée}

Comme l'accès à un travail stable, l'indépendance résidentielle marque une étape décisive dans le processus qui conduit à l'autonomie pour tous les jeunes, qu'ils aient été ou non placés ${ }^{6}$. Rien ne traduit mieux le passage au statut d'adulte pour les jeunes anciennement placés que vivre de manière indépendante, «chez soi », ni dans un foyer, ni dans un appartement relais avec supervision.

Très vite à la sortie du VE, un tiers des jeunes ont réussi à avoir un logement indépendant. Environ un sur cinq vit soit dans les appartements destinés aux jeunes du VE, soit en foyers (foyers Jeunes Travailleurs..), et une proportion identique est hébergée par la fratrie, de même par la mère SOS. Quelques uns vivent chez des amis; les retours dans la famille d'origine sont minoritaires $(6 \%)$ et ils concernent surtout les sorties précoces avant l'âge de 17 ans. Dès le deuxième logement, l'indépendance résidentielle est acquise par six sujets sur dix, à égalité entre hommes et femmes (tableau 1). Dès cette étape, l'appartenance générationnelle différencie les parcours d'accès au logement individuel : au deuxième logement, près des trois quarts des «anciens » ont déjà acquis une indépendance résidentielle vs la moitié seulement pour les plus jeunes. Par ailleurs, les sujets diplômés accèdent plus rapidement à une autonomie résidentielle ; la moitié des sans diplômes n'ont pas encore de logement individuel dans les premières années qui suivent la sortie. Enfin, l'étude des logements successifs (reconstruction des trajectoires résidentielles) montre que les hommes sont 32\% vs 3\% pour les femmes) à ne pas avoir encore de logement individuel à 25 ans, quelle que soit la période à laquelle ils sont sortis.

\section{Les parcours d'insertion problématiques}

Au moins $15 \%$ des sortants, parmi ceux ayant répondu à l'enquête postale, ont rencontré, de manière temporaire, des problèmes avec la justice et la police, et/ou des épisodes de violence, de consommation de psychotropes. Par ailleurs, quelques uns ont en plus été suivis

\footnotetext{
${ }^{6}$ En France, l'âge médian de l'accès à l'indépendance financière résidentielle a peu changé entre 1992 et 1997 , date à laquelle il était de 22,8 ans pour les hommes et 21,5 ans pour les femmes (Villeneuve-Gokalp, 2000).
} 
et ont bénéficié d'un accompagnement par des travailleurs sociaux dans les premières années de la sortie. Ces parcours concernent deux fois plus les hommes que les femmes; pour ces dernières, on peut supposer qu'une insertion conjugale rapide leur a vraisemblablement permis d'éviter de s'engager dans une trajectoire de « galères ».

\section{Des solidarités familiales très présentes}

Quelle que soit l'époque, ces jeunes ne peuvent compter sur un soutien affectif ou matériel de leurs parents disparus ou toujours dysfonctionnants. Quelques tentatives de retour en famille d'origine à la fin de la prise en charge se sont souvent soldées par des déceptions, quand elles n'ont pas engendré de nouveaux traumatismes pour le jeune (famille incestueuse, violente). Dans les premières années qui suivent la sortie, outre les aides institutionnelles, $59 \%$ des jeunes ont pu bénéficier de soutiens financiers et matériels de leur environnement proche. Pour les générations anciennes (fratries nombreuses et très souvent orphelines), cette solidarité provient essentiellement des frères et sœurs aînés, ayant ou non vécu au VE, alors que c'est principalement la mère SOS pour les jeunes générations. D'une manière générale, ce sont les hommes qui ont le plus bénéficié de soutiens familiaux (tableau 1). En effet, l'hébergement par la mère SOS s'adresse plus à ces derniers (période entourant le service militaire) ; les femmes, quant à elles, rencontrent plus rapidement un conjoint avec qui elles cohabitent. Il est notable de constater que, même en cas de troubles psychiques ou comportementaux, cette solidarité familiale fonctionne : il n'y a en effet aucune différence significative dans l'aide apportée par les frères et sœurs ou la mère SOS selon l'existence ou non de troubles.

\section{Discussion et perspectives}

A la différence des autres institutions de placement, les villages d'enfants offrent des accueils de longue durée pour des fratries. Le propos de cet article est d'analyser les parcours d'accès à l'indépendance vers l'âge de 25 ans d'anciens placés dans un village d'enfants SOS. L'étude présente un double atout : l'observation de plusieurs générations de sortants et un recul temporel important depuis la fin des prises en charge. Ainsi ont pu être prises en compte les évolutions institutionnelles des accompagnements des jeunes et les transformations des contextes socio-économiques en termes d'insertion professionnelle.

Il y a une trentaine d'années, les soutiens institutionnels à la sortie du VE étaient minimes, les sortants ne disposaient alors que d'aides individuelles de la part de leurs frères et sœurs et de leur mère SOS. Néanmoins, ils ont bénéficié, au moment de leur sortie, d'un marché de l'emploi favorable, même en l'absence de qualification importante. Par la suite, les sortants ont vécu des changements dans la politique scolaire comme, par exemple, le processus d'allongement des durées de formation initiale et d'élévation des niveaux d'études dans le secondaire, ce qui peut expliquer la plus forte proportion de non diplômés parmi eux. C'est essentiellement à partir des années 1980, avec la mise en place de l'équipe socio-éducative, que les aides institutionnelles se sont développées et diversifiées. De tels supports se sont révélés d'autant plus nécessaires que ces jeunes ont été confrontés à une dévalorisation des diplômes de petit niveau (CERC, 2008) et à la dégradation des conditions d'entrée sur le marché du travail. Ainsi, malgré toutes ces aides, on note un décalage entre les niveaux d'études atteints et l'obtention des diplômes correspondants, pour près de la moitié des jeunes, qu'ils aient ou non des problèmes de santé mentale.

Comme dans d'autres études (Trout, Hagaman, Casey, Reid \& Epstein, 2008), les jeunes sont plus nombreux que dans la population générale à n'avoir aucun diplôme : 40\% vs 15\% ; ils sont aussi plus nombreux à manifester des problèmes psychiques et comportementaux : $40 \%$ vs $12 \%$ (INSERM, 2002). Ces troubles vont non seulement retentir sur le cursus scolaire 
mais aussi sur le cheminement ultérieur en terme d'accès à un emploi qualifié et à un logement stable. Plus que l'existence de ces problèmes, c'est leur degré de gravité, et notamment leur pérennité, qui déterminent les chances d'accéder à l'indépendance.

Comparativement à d'autres institutions, une des spécificités des villages d'enfants tient à l'importance des dispositifs d'aides après la sortie, parfois même au-delà de l'âge de 21 ans, aides institutionnelles et également aides personnelles des accueillantes, les mères SOS.

C'est grâce à ces soutiens prolongés qu'un quart des jeunes ont obtenu un diplôme et que nombre d'entre eux, dont certains très en difficultés, sont parvenus à s'insérer sur le plan de l'emploi et du logement. Par ailleurs, déjà signalée par d'autres études sur les sorties des placements, l'instabilité résidentielle, faite de nombreux déménagements et d'hébergements provisoires, est nettement plus fréquente chez les hommes que chez les femmes.

Depuis quelques années, de tels dispositifs d'aides sont largement préconisés aux EtatsUnis et au Canada (Courtney \& Downey, 2006 ; Goyette, 2006 ; Vacca, 2008). C'est aussi grâce à eux que, pour de nombreuses femmes, la formation et l'emploi ont constitué les premières étapes de l'autonomisation, avant la constitution du couple et l'entrée dans la maternité. Au total, neuf jeunes sur dix ont accédé, plus ou moins facilement, à l'indépendance à l'âge de 24-25 ans. Cette fréquence élevée renvoie à la spécificité de la structure Villages d'Enfants : stabilité du placement en VE, importance des prises en charge institutionnelles et implication des mères SOS pendant la durée du placement et bien au-delà.

Le contexte de professionnalisation des mères SOS, aujourd'hui " éducatrices familiales », conduit à s'interroger sur le devenir de leurs relations avec les jeunes qu'elles auront accueillis. S'il est sûr que des attachements électifs se maintiendront de part et d'autre, qu'en sera-t-il des supports et des soutiens concrets qu'elles prodigueront à long terme ?

Comme dans tous les dispositifs de placement, la durée de placement en villages d'enfants tend à se réduire, les enfants qui arrivent plus jeunes ont déjà vécu des placements antérieurs, ils restent moins longtemps et retournent davantage dans leur famille. L'ensemble de ces tendances, contribuant à une moindre stabilité de vie des enfants, renforce la nécessité d'une réflexion réelle autour d'actions de soutien innovantes. Les situations de retours en famille qui fragilisent les jeunes sur le plan psychoaffectif devraient s'inscrire dans un dispositif d'accompagnement spécifique par l'institution elle-même ou dans une action coordonnée avec les autres partenaires sociaux. L'importance de tels supports relationnels auprès des jeunes, en particulier ceux de 15 ans et plus a été d'ailleurs soulignée (Biehal, N., Clayden, J., Stein, M. \& Wade, 1995 ; Osterling \& Hines, 2006 ; Wade, 2008).

Afin de favoriser l'estime de soi et de faciliter la transition vers la vie adulte, quelques interventions plus adaptées commencent à être mises en pratique Outre Manche auprès des adolescents accueillis dans les structures de la Protection de l'enfance (Lemon, Hines \& Merdinger, 2005 ; Goyette, 2006). En France, de nouveaux dispositifs départementaux sont expérimentés auprès de jeunes de 16 à 21 ans confiés à l'ASE et la PJJ, visant à leur éviter des parcours de marginalité et d'exclusion. Il reste que la préoccupation des équipes socioéducatives gagnerait à se centrer précocement sur le scolaire - aides dans le choix des orientations professionnelles, encouragements à aller jusqu'à l'obtention d'un diplôme - dans la finalité d'une entrée rapide dans le monde du travail mais également dans la perspective d'une plus grande souplesse et ouverture des trajectoires professionnelles à long terme.

En final, toutes les formes d'interventions, depuis la socialisation au quotidien avec l'éducatrice familiale aux accompagnements institutionnels et à leurs partenariats extérieurs, demandent à s'inscrire dans un projet cohérent de préparation précoce à l'autonomisation et à l'accès au statut d'adulte. 


\section{Remerciements}

Cette partie du programme de recherche a fait l'objet d'une convention d'étude avec la DGAS du Ministère de l'emploi, de la cohésion sociale et du logement, et du Ministère de la santé et des solidarités. Elle a également été financée par l'Association SOS Villages d'Enfants. Par ailleurs, les auteurs remercient aussi les psychologues Marie Constantin-Kuntz et Catherine Mousseau-Stella qui ont mené une partie des entretiens avec les anciens placés.

\section{Références bibliographiques}

Andersson, G. (2005). Family relations, adjustement and well-being in longitudinal study of children in care. Child and Family Social Work, 10, 43-56.

Bailleau, G., Trespeux, F. Les bénéficiaires de l'aide sociale départementale en 2007. DRESS, Etudes et Résultats, ${ }^{\circ} 656$, septembre 2008.

Bauer, D., Dubechot, P., \& Legros, M. (1993). Le temps de l'établissement : des difficultés de l'adolescence aux insertions du jeune adulte. Paris : CREDOC.

Biehal, N., Clayden, J., Stein, M. \& Wade, J. (1995). Moving on: Young people and leaving schemes. Her Majesty's Stationery Office, London.

Caille, J. P. (2003). L'école réduit-elle les inégalités sociales ? Education et formations, 66, 177-185.

Carpenter, S. C., Clyman, R. B., Davidson, A. J., \& Steiner, J. F. (2001). The association of foster care or kindship care with adolescent sexual behavior and first pregnancy. Pediatrics, 108(3), p. e46.

Conseil de l'Emploi, des Revenus et de la Cohésion Sociale. (2008). L'insertion des jeunes sans diplôme. Rapport CERC $\mathrm{n}^{\circ}$ 9. Paris : La Documentation Française.

Coppel, M.\& Dumaret, A.C. (1995) Que sont-ils devenus ? Les enfants accueillis à l'Oeuvre Grancher. Analyse d'un placement familial spécialisé. Ed. Erès, Ramonville St Agne.

Corbillon, M., Duléry, A., \& Mackiewicz, M. P. (1997). Quel devenir à l'issue d'un placement dans une maison d'enfants? (Rapport final). Orléans: GERIS.

Courtney, M., Piliavin, I., Grogan-Kaylor, A., \& Nesmith, A. (2001). Foster youth Transitions to Adulthood: A Longitudinal View of Youth Leaving Care. Child Welfare, 6, 685-717.

Courtney, M. E., \& Dworsky, A. (2006). Early outcomes for young adults transitioning from out-of-home care in the USA. Child and Family Social Work, 11, 209-219.

Davaillon, A., \& Nauze-Fichet, E. (2004). Les trajectoires scolaires des enfants 'pauvres'. Education \& Formations, 70, 41-63.

Del Valle, J. F., Bravo, A., Alvarez, E., \& Fernanz, A. (2008). Adult self-sufficiency and social adjustment in care leavers from children's homes: A long-term assessment. Child and Family Social Work, 13, 12-22.

Dumaret, A. C., Coppel-Batsch, M., \& Couraud, S. (1997). Adult outcome of children reared for long term period in foster families. Child Abuse and Neglect, 21, 911-927.

Dumaret, A. C., Crost, M. (2008). Placements à long terme en villages d'enfants depuis plus de 30 ans : troubles psychiques et prises en charge. Neuropsychiatrie de l'Enfance et de l'Adolescence, 56, 512-519.

Festinger, T. (1983). No one ever asked us. A postcript to foster care. New-York: CUP.

Frechon, I. (2005). Stratégies féminines d'entrée dans la vie adulte après un placement à l'adolescence ». In E. Callu, J. P. Jurmand, \& A. Vulbeau, La place des jeunes dans la cité (pp. 215-232), Tome 2, Paris : L'Harmattan. Espaces de rue, espace de parole. 
Frechon, I., \& Dumaret, A. C. (2008). Bilan critique de 50 ans d'études sur le devenir des enfants placés. Neuropsychiatrie de l'Enfance et de l'Adolescence, 56, 135-147.

Gaudet, S. (2007). L'émergence de l'âge adulte, une nouvelle étape du parcours de vie. Implications pour le développement de politiques. Document de discussion. Canada: Projet du PRP.

Goyette, M. (2006). Préparation à la vie autonome et l'insertion socioprofessionnelle des jeunes pris en charge par l'Etat: quelles interventions? Revue Sociétés et jeunesses en difficulté. On line : http://sejed.revues.org/document159.html.

Hubert, T, Tournyol du Clos, R., Cosio, M., \& Frechon I. (2006). Le devenir des jeunes pris

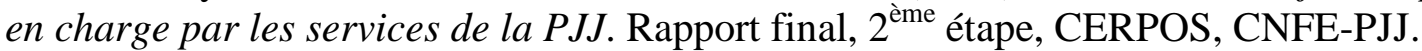

INSERM, Expertise collective (2002). Troubles mentaux. Dépistage et prévention chez l'enfant et l'adolescent. Paris: Inserm Eds.

Lemon, K., Hines, A. M., \& Merdinger, J. (2005). From foster care to young adulthood: The role of independent living programs in supporting successful transitions. Children and Youth Services Review, 27, 251-271.

Lenz-Rashid, S. (2008). Employment experiences of homeless young adults: Are they different from youth with a history of foster care? Children and Youth Services, 28, 235259.

Osterling, K. L., \& Hines, A.M. (2006). Mentoring adolescent foster youth: promoting resilience during developmental transitions. Child and Family Social Work, 11, 242253.

Pecora, P. J., Kessler, R. C., O'Brien K., et al. (2006). Educational and employment outcomes of adult formerly placed in foster care: Results from the Northwest Foster Care Alumni. Children and Youth Services Review, 28, 1459-1481.

Pinkerton, J., \& Stein, M. (1995). Responding to the needs of young people leaving state care: Law, practice and policy in England and Northern Ireland. Children and Youth Services Review, 5/6, 683-695.

Rashid, S. (2004). Evaluating a transitional living program for homeless, former foster care youth. Research on Social Work Practice, 14, 240-248.

Reid, C., \& Dudding P. (2006). Building a future together: Issues and outcomes for transition-aged youth, Centres of excellence for children's well-being, Ottawa, 58 p.

Rutter, M., Quinton, D., \& Hill J. (1990). Adult outcome of institution-reared children: Males and females compared », In L. Robins (Eds.) Straight and devious pathways from childhood to adulthood (pp. 135-157). Cambridge: Cambridge University Press.

Stein, M. (1994). Leaving Care, Education and careers Trajectories. Oxford Review of education, 20(3), 349-360.

Stein, M. (2006). Research Review: Young people leaving care. Child and Family Social Work, 11, 273-279.

Stein, M., Munro, E. R. (Eds.). (2008). Young people's transitions from care to adulthood: International research and practice (pp. 49-62). London: Jessica Kingsley Publishers.

Stor $\varnothing$, J. (2008). Exit from care-developing a perspective, Journal of Comparative Social Welfare, 24(1), 13-21.

Trout, A. L., Hagaman, J., Casey, K., Reid, R., \& Epstein, M. (2008). The academic status of children and youth in out-of-home care: A review of the literature. Children and Youth Services Review, 30, 979-994.

Vacca, J. S. (2008). Foster children need more help after they reach the age of eighteen. Children and Youth Services Review, 30, 485-492.

Villeneuve-Gokalp, C. (2000). Les jeunes partent toujours au même âge de chez leurs parents. Economie et Statistiques, 337/338, 61-80. 
Article paru dans La Revue Internationale d'Education, n²6, 2009, 15-34

Wade, J., \& Dixon, J. (2006). Making a home, finding a job: investigating early housing and employment outcomes for young people leaving care. Child and Family Social Work, $11,199-208$.

Wade, J. (2008) The ties that bind: support from birth families and substitute families for young people leaving care. British Journal of Social Work, 38, 39-54. 
Article paru dans La Revue Internationale d'Education, n²6, 2009, 15-34

Tableau 1 : Principales caractéristiques des jeunes accueillis en VE et accès à l'indépendance selon le genre.

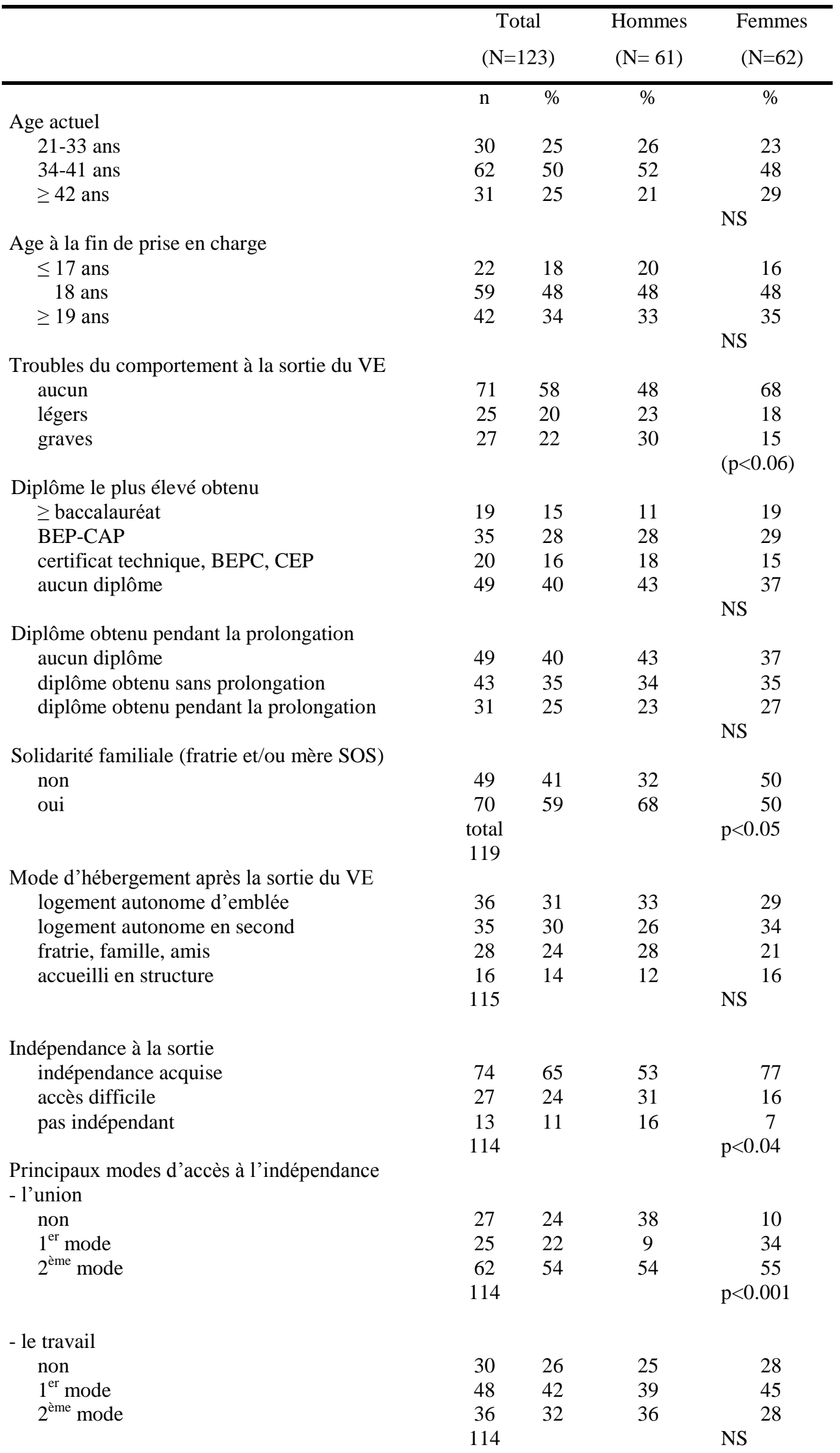


Article paru dans La Revue Internationale d'Education, n²6, 2009, 15-34

- les petits boulots

$1^{\mathrm{er}}$ mode

$2^{\text {ème }}$ mode

$69 \quad 61$

48

72

$\begin{array}{llll}35 & 31 & 45 & 17\end{array}$

$\begin{array}{llll}10 & 9 & 7 & 10\end{array}$

$114 \quad \mathrm{p}<0.01$ 
Article paru dans La Revue Internationale d'Education, n²6, 2009, 15-34

Fig.1 Aides institutionnelles au moment de la sortie, selon l'âge.

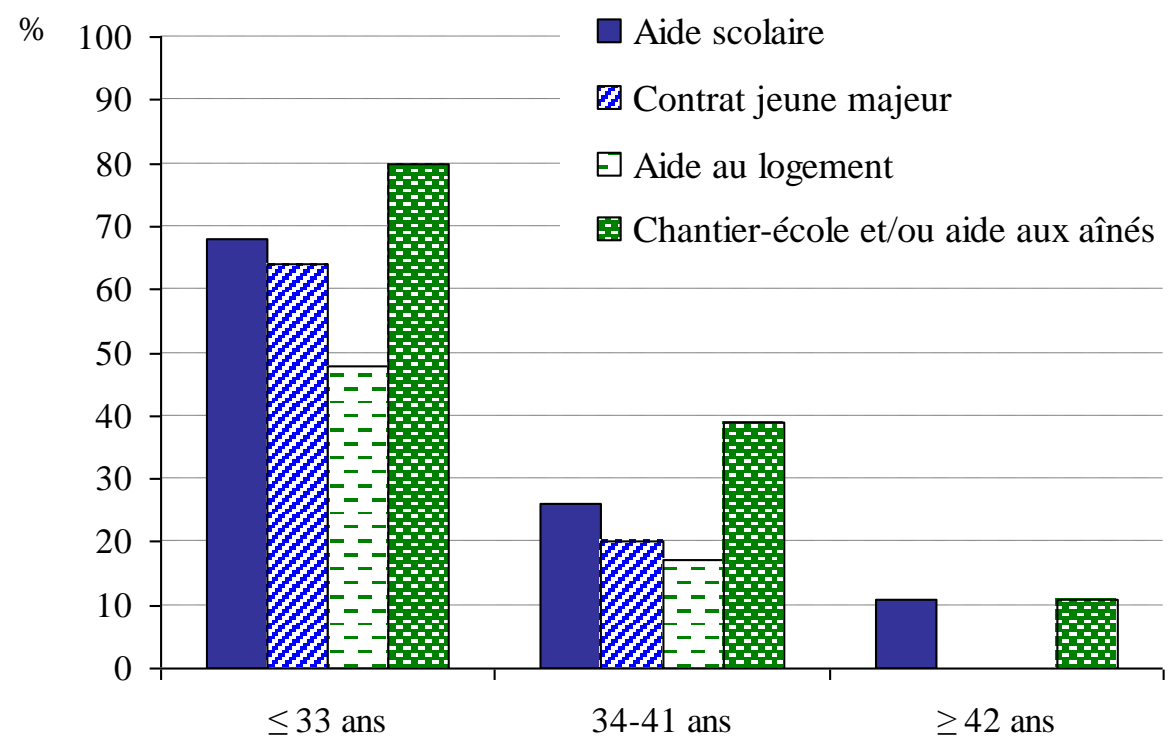

Fig.2 Accès à l'indépendance selon l'âge et troubles psychiques et comportementaux à la sortie.

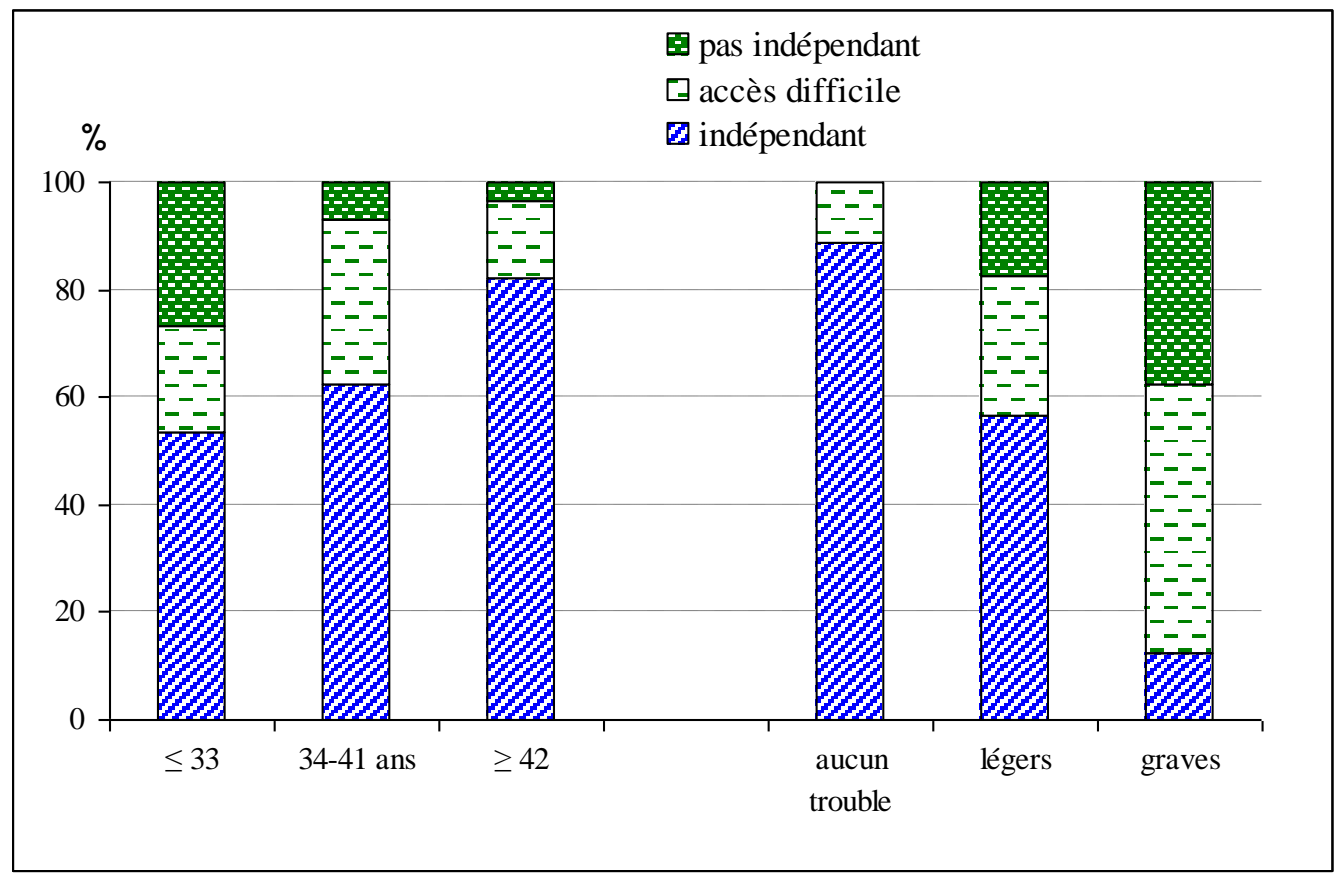

